\title{
The Design and Development of an Automatic Construction Crane*
}

\author{
M. Abderrahim, A. Giménez, A. Nombela, S. Garrido, R. Diez, V. \\ M. Padrón and C. Balaguer
}

\author{
Department of Electrical Engineering, Electronics and Automation \\ Universidad Carlos III de Madrid \\ C/Butarque, 15, 28911 Leganés, Madrid (Spain) \\ e-mail: \{mohamed, balaguer\}@ing.uc3m.es
}

\begin{abstract}
This paper describes the design and development of an automatic crane for use in the assembly of modular buildings elements in the construction site. The automatic system was developed using a commercial crane, which was modified by adding the adequate sensors, servomotors and an advanced control system. The new crane system is meant to work in an automatic or semi-automatic manner to achieve the assembly of modules following the sequence established during the design process. The assembly path shall be readjusted to compensate for the deviations between the planned and actual loading positions of the modules to be assembled. A special handling platform was designed to allow the grasping of the modules via electromagnets and female conical locators/connectors. The male conical connectors are part of building modules themselves, and are also used to ensure the connection between modules.

The cranes system is controlled via computer using a multi-axes control board implementing adequate algorithms to ensure a smooth movement and prevent the undesired swinging effects. The system is operated via a Man Machine Interface (MMI) installed in the control computer, which make use of new features as well as via the traditional push button pendant used in commercial cranes. Although the system is currently functional, new features such as the security system and the image processing modules are still under development.
\end{abstract}

Keywords: crane automation, construction automation, modular building.

\section{INTRODUCTION}

Traditional methods on house building are usually based on manual techniques, which are slow and expensive. The benefits of construction industries can be improved by using advanced manufacturing systems. These systems will increase the quality and the customer satisfaction. Nowadays, other industries take advantage of automatic production and assembly processes. Despite that the research to develop new construction techniques has been important during the past few years [1], there is still an important gap between the construction industry and other industries.

Automation is reaching different levels of the construction industry, in non-uniform manner, due to the nature of each project and site. Successful efforts have been made to introduce automation in the production of prefabricated facade panels [2] [3], and some manufacturers already make 3D building modules on an assembly line [4]. This represent the future of the construction industry, where parts of the building come as finished product from the factory, which entails a shorter labour effort at the building site. This time can be minimised further if automatic assembly techniques are introduced at this side. Exchange of information between the different stages (design, planning, transport, erection, maintenance, etc.) is a very valuable asset to bring automation into the construction industry [5].

The work described in this paper focuses on the last part, which consists of the automation of the assembly of prefabricated building elements. The system was developed using a commercial crane, which was modified by adding the adequate sensors,

\footnotetext{
* Partially supported by FutureHome project (BRITE nº ER4-29671)
} 
servomotors and an advanced control system. The adapted crane can be used as a traditional machine, which is operated by a hand-held push button pendant, as well as in automatic mode. In the latter mode the handled parts " prefabricated modules" are taken from their loading locations to their final positions in the building through a pre-calculated path, which avoids obstacle and already assembled parts of the building.

The rest of the paper describes the different elements of the automated crane.

\section{CRANE DESIGN}

\subsection{The Crane Structure.}

The size of the crane to undergo adaptation had to be decided taking into account the assembly tests to be performed and the available resources including the space. Due to the impracticality of using real size 3D prefabricated building modules we opted for preparing $1 / 3$ scaled model of these elements with the main constituents (structure and connectors). It has been though practical to use a small size Gantry crane to fit in the laboratory space. Assembly simulation tests were used to select the size and characteristics of the crane structure, figure 1.

$$
\begin{array}{ll}
\text { Span } & 4 \mathrm{~m} . \\
\text { Payload } & 500 \mathrm{~kg} .
\end{array}
$$

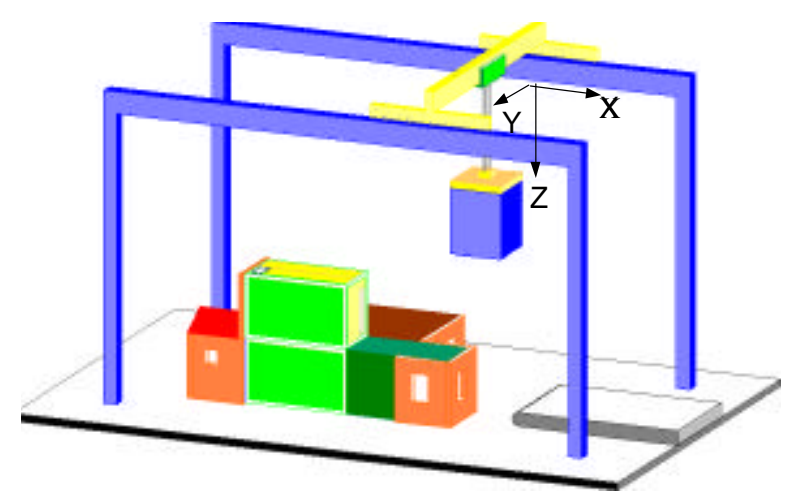

Figure 1. Drawing of the gantry crane taken from the simulation

The purchased conventional crane has been stripped from its conventional motors and wiring in order to adapt it by including the adequate servomotors and controls.

\subsection{Crane Conversion.}

The crane have been fitted with new equipment to make it function similar to a Cartesian robot manipulator. New electrical servo motors have been selected to replace the old ones. All motors are fitted with a position sensors (3 resolvers and encoder). Two motors drive the crane bridge through reduction gears ending in graphite treated ductile cast iron wheels, which move on top of steel rail.

The trolley supports the hoisting motor, the drum and the trolley motor. The trolley slide on the main beam of the bridge (wide flange H-profile beam).

All newly added motors are AC brushless servomotors fitted with planetary gear, brake and resolver. These servomotors are coupled to the end trucks and trolley via steel made couplings.

The original hoist motor was kept. This motor comes with gear, brake and security sensors. An incremental encoder has been added to allow the position control of this axis. The drum supports the mechanical cable of the crane.

\subsection{Motors and Control Sensors}

In addition to position sensors mentioned above, more sensors and safety devices were fitted to the crane. Limit switches were fitted in each movement axis of the crane to prevent handling objects beyond these points. To ensure more security, about ten centimetres after the limit switches mechanical blocking mechanisms were installed, with rubber buffers to avoid direct collision between metal parts.

To measure swinging of the load an inclinometer was installed on top of the handling platform, which will be described later. The device measures the inclination angle about two axes. This information will be used to reduce the load swinging using the control system.

The motion of the crane joints is ensured by the described motors and adequate drivers; one for each motor. These drivers receive the control signals from the multi-axes control board installed in a PC and produce the corresponding Power to make the motors move according to orders. These power drive are based on PWM technology. They can vary the motor speed to achieve fine acceleration and deceleration and can give maximum torque at zero speed.

\subsection{Joint Control Board}

The motion control is guaranteed by a commercial multi-axes control plug-in board. The selected board is the PMAC-PC board from Delta Tau Data Systems. It is one of the most powerful, most versatile and cost effective motion controllers according to the manufacturer description. It is able of handling up to 8 simultaneous axes. The PMAC's speed response and power allow it to handle complex motion trajectories, difficult servo filters, advanced computation algorithms, and sophisticated I/O logic simultaneously. The PMAC board and control software are hosted in the same PC; designated Control PC. Among other, software the PC run the program that controls the joint motion. Figure 2 shows the main motion and control hardware 


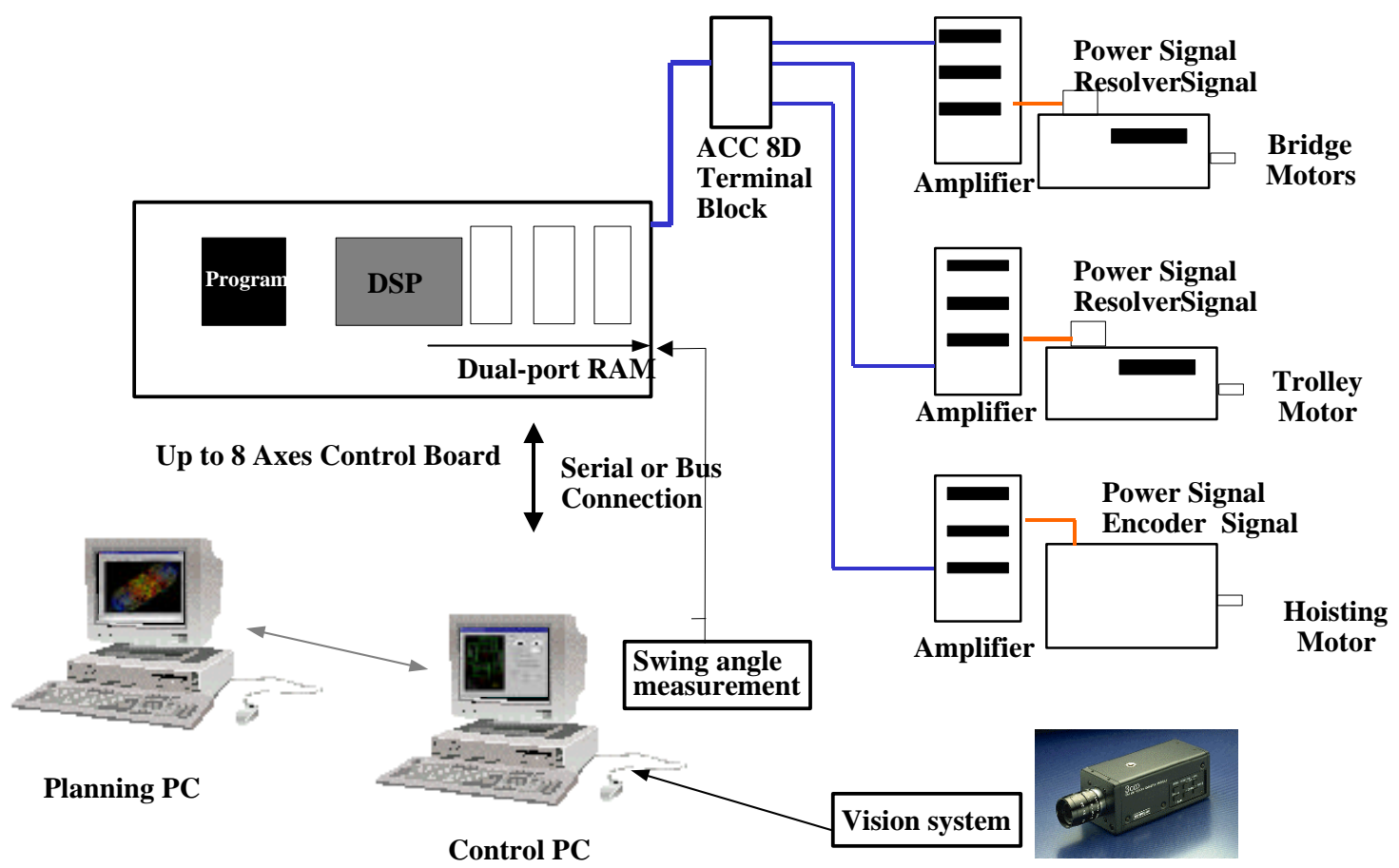

Figure 2: General hardware architecture for motion control applications

interconnections in addition to other details as explained bellow.

In addition to the motion control hardware it was necessary to include more sensors and devices to increase the security and simplify the operation of the automatic system as mentioned above. The information from limit switches is fed back to the control board to prevent motion beyond the accepted limits. The information from the inclinometer is read by the computer via the serial port and a program that extract the swing angles of the crane cables. Those angles are used then by the control algorithm.

As shown in figure 2 The control PC also hosts a frame grabber, which receives the image from four cameras located at adequate location of the crane to monitor and aid the positioning of the load.

\section{SOFTWARE}

The use of Windows was recommended because of the software support to part of the used hardware and for reasons operation ease. The control and operation software was designed in way to allow the crane to be operated manually or in an automatic manner. The programmes were written in the $\mathrm{C}++$ language as separate modules, each responsible for a given function. These modules are linked to complement each other and perform the task of control and monitoring. All sensor reading are made via sent to main program via their corresponding modules (drivers); see figure 3 . The main program is the link between the majority of these routines. The operator interact with the crane system via a Graphical Man Machine Interface (MMI or GUI), coded as part of the main program.

The main program calls and executes the other modules when necessary. For instance, if a given movement is required, The actual joint positions are read and an adequate trajectory profile is calculated for each joint then using the instant position and the inclinometer information through the serial port, the used algorithm generate the control signal and send it to the control board. The latter ensures moving the motors via the power drivers. The control board monitor several data and signals while moving the motors. If the emergency button is pressed all movements are halted. The frame grabbing module on the other hand is responsible for capturing the image from the cameras and showing them on screen of the operator. There is an image processing modules which looks for some predefined features to locate building modules or parts in the site. Although this module is not finalised at the time of writing this paper, initial results are satisfactory.

\section{CRANE OPERATION}

As mentioned in the previous section a graphical MMI is main interaction tool between the operator and the automated crane. However, the system is designed to function as a traditional crane fitted with the handling platform, described in next section. 


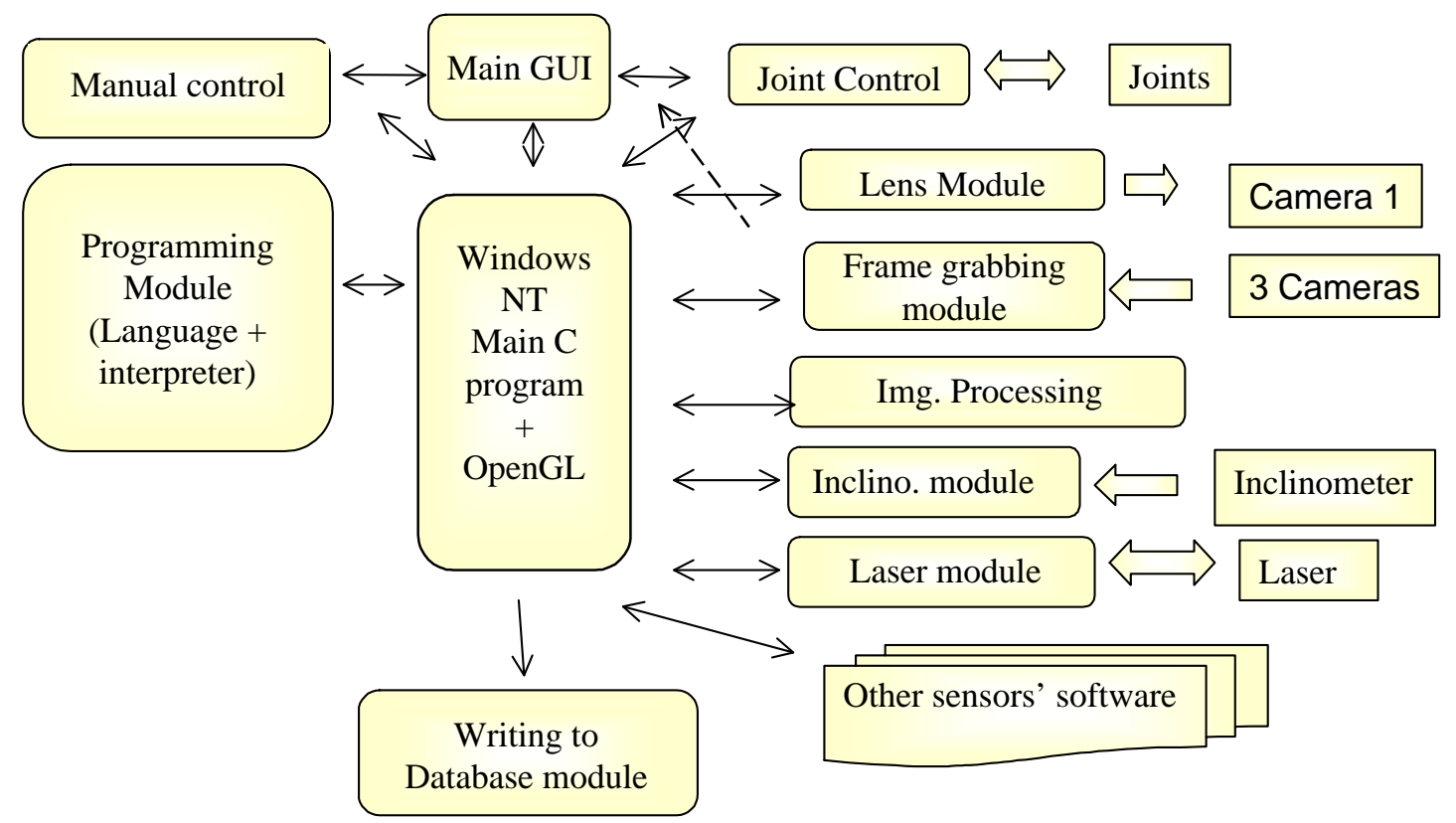

Figure 3: General software architecture for control and operation of the system

\subsection{The Automatic Mode}

During the automatic mode the crane is able to move minimum intervention from the operator, following trajectories read from the database of the project in hand. When parts and building are moved to their required final positions, this information can communicated to the database. This information permits to know instantly the current progress of the assembly and the project as a whole.

Sequence of movement can also be coded via the programming editor provided as part of the MMI, Figure 2. The recommended operation mode is the semi automatic, where most of the assembly task is programmed but confirmation from the operator is required during the handling key situations.

The MMI is to provide the user visual information about the state of various elements of the crane and useful tools and features as listed bellow:

1. a menu/tool bar for choosing program files, saving, editing, configurations, help ...etc.

2. Mode (automatic/manual),

3. a graphical hand-held like pendant for manual mode,

4. all sensors status (values),

5. the 3 cameras' views, optional

6. an alarm icon,

7 a halt button,

8. a programming window,

9 The status of the joints (actual values).

10. a tool for saving the accomplished steps for database use and more features that may be useful for the project being executed.
Command line for executing programs such as:

- Motion programs for the PMAC control board

- PLC programs for the PMAC control board

- Drivers for the sensors like inclinometer, cameras

- Image processing software for the vision system

\subsection{The Manual Mode}

There are two distinct manual modes that can be used when operating the developed crane. The first, can be performed via graphical handheld-like pendant, which moves the crane along the axis which button is pushed on the screen. The second, is performed via a physical push button pendant which ensure the movement of all axes just like a traditional crane. In case the pendant supplies a constant reference to the power drive, which can interpreted as a command to move the motor in a given direction, after releasing the brakes of the commanded motor.

At the time of writing this paper the MMI was not complete and several of the movement programs were executed through command lines.

\section{HANDLING PLATFORM}

As described earlier, the original hook of the gantry crane was replaced by a platform for the handling and assembly of modular blocks.

The platform consists of a square skeleton, 800 $\mathrm{mm}$ a side, joined in the centre point to the pulley group of the gantry crane; figure 4 and 5 . The axis 
joining the platform and the pulley group is designed to allow rotation for the later inclusion of controlled movement in this axis.

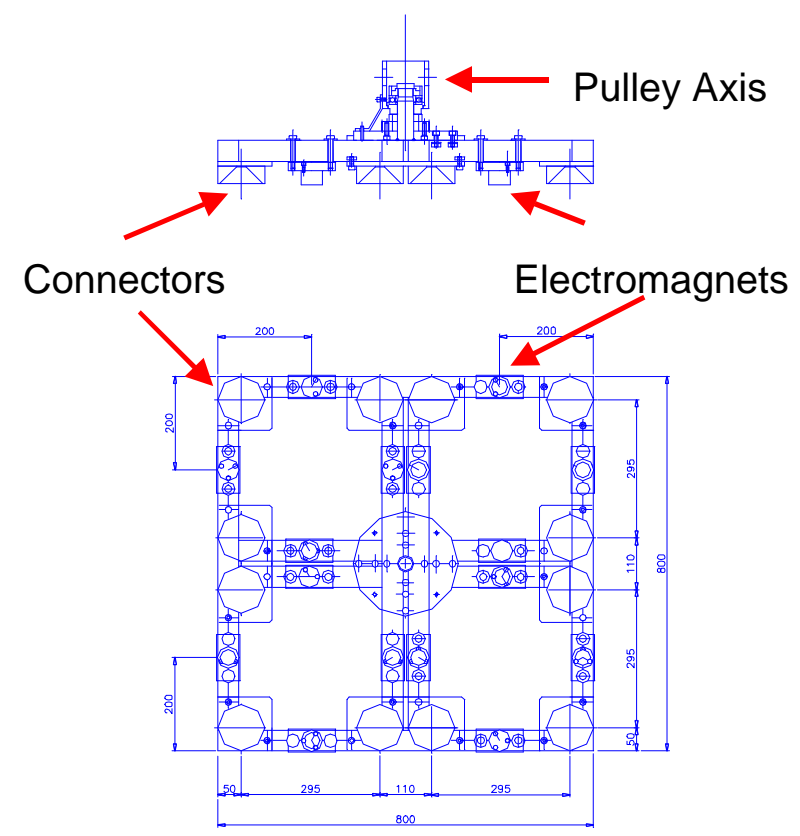

Figure 4: The Handling Platform drawing

Since the platform is developed for the handling of specifically designed and built elements such as the prefabricated building blocks (3D and 2D facade elements), it is equipped by adequate sensors and grasping tools.

The platform carries various female parts of autocentring conical connectors. When the platform is lowered on top of the modules to be handled, which carry the male side of those connectors, it adjusts automatically in the right position under its own weight.

The platform contains 16 electromagnets to capture the modules. They are uniformly distributed to avoid platform unbalance and to support the module weight. When these electromagnets are powered they attract the module. Then, the crane moves it to its corresponding location on the

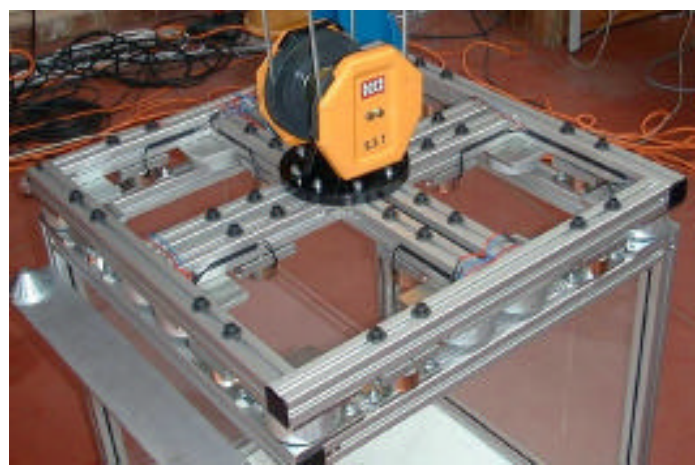

Figure 5: The Handling Platform structure of the building. Next, the module is lowered until the male connector in the floor platform and the female connector in the bottom of the module are connected.

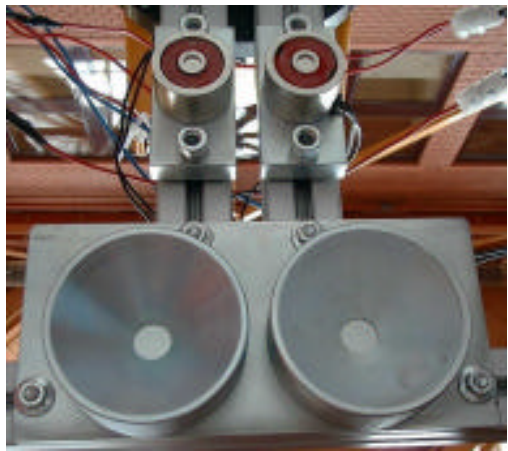

Figure 6: Electromagnets and connectors in the platform base

The electromagnets are all powered together by DC voltage. The electromagnets in the perimeter of the platform are arranged to support the payload of 3D modules (single or double), while the electromagnets in the centre of the platform are arranged to support the payload of a $2 \mathrm{D}$ or vertical modules. Figure 6 shows 2 of the 16 electromagnets in base of the platform. When all electromagnets are powered, the platform is able to handle modules of up to $160 \mathrm{~kg}$.

In addition to the conical connectors and electromagnets the platforms curries other sensors. There are 4 cameras fixed to the platform that can capture the images from under the platform and send them to the control PC for visualisation and control purposes. The inclinometer is also fixed on top of the platform in a central position to measure swinging angles.

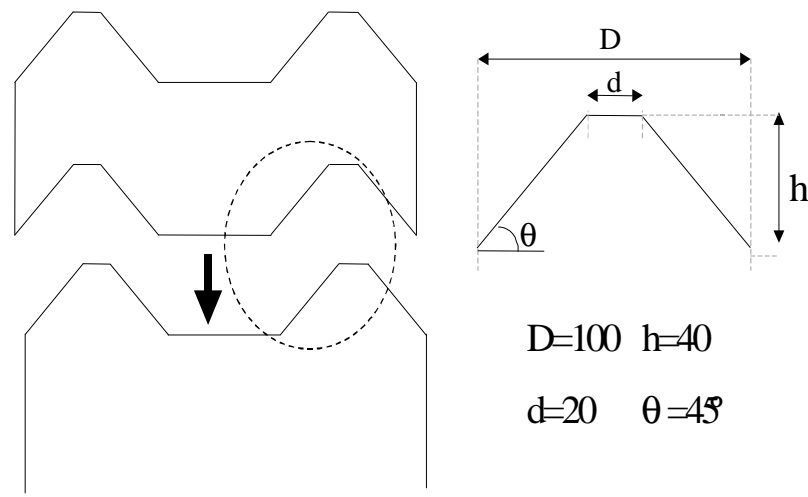

Figure 7: Connectors geometry

The connectors, both male and female members were chosen of conical form to ensure the autocentring and compensation of small positioning errors between modules and between handling platform and the handled module during assembly operations. 


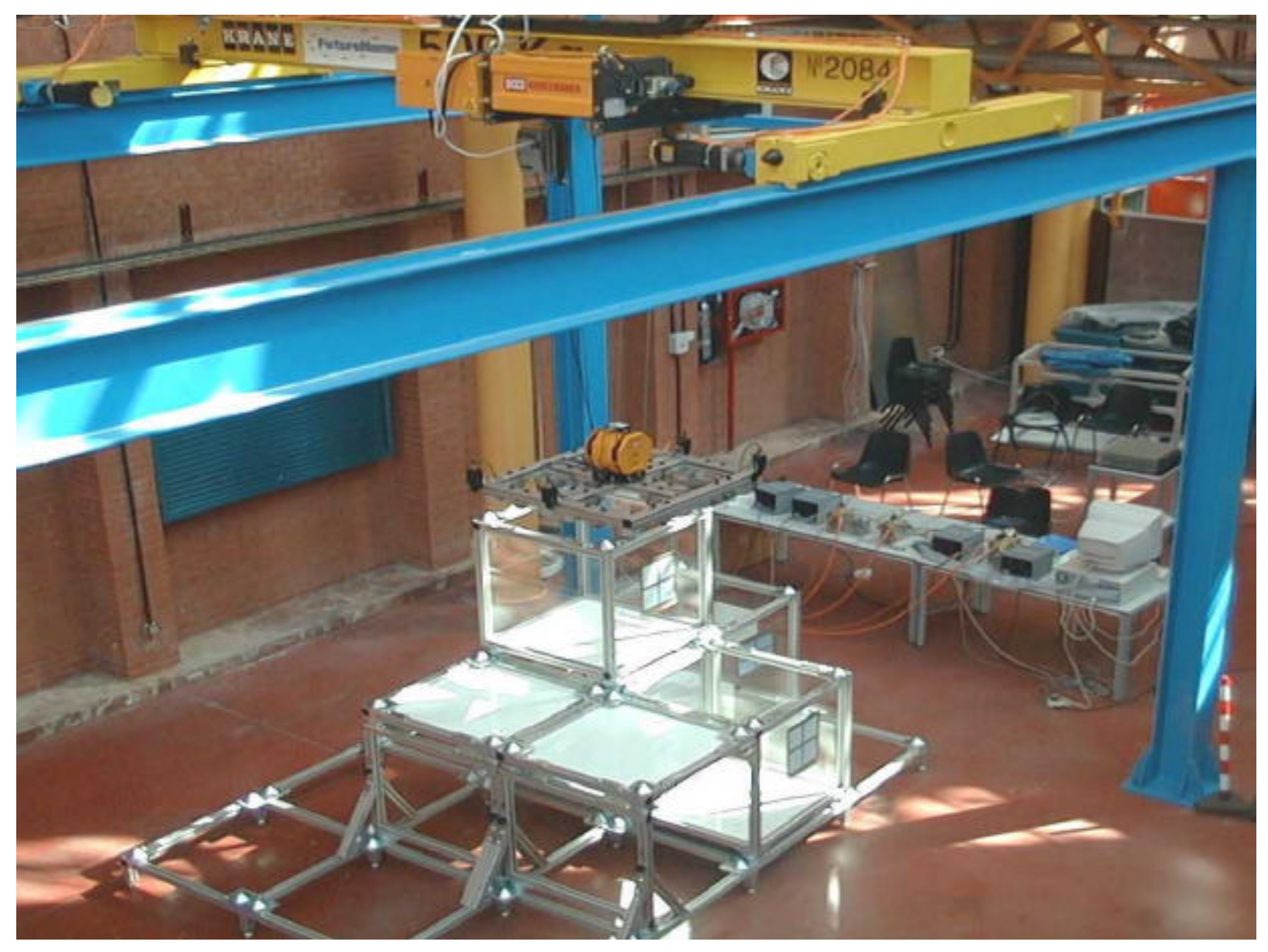

Figure 8: A general view of the automated crane in the lab

For connectors made of soft steel the inclination angle to allow sliding of the cones has to be at least $30^{\circ}$. For the model used in our laboratory the chosen angle is $\mathrm{e}=45^{\circ}$. With this angle and the values given to the parameters $\mathrm{D}$ and $\mathrm{d}$ (see figure 7 ) the positioning tolerance is $(\mathrm{D}-\mathrm{d}) / 2$, which is $4 \mathrm{~cm}$. This means that if the crane reaches the desired positions with $\pm 4 \mathrm{~cm}$ error the modules will be assembled correctly since the connectors themselves shall centre the modules bringing them into the correct position under their proper weight.

\section{CONCLUSIONS}

The developed automatic crane (figure 8) presents a new tool to close the link between manufacture of the prefabricated buildings' modules and the assembly on site using automated means. The outcome demonstrates the feasibility of the process of automation and shows how the system could assemble 3D and 3D modules with the help of the designed handling platform. Precision and safety features are incorporated in the system to minimise accidents and damage to humans and machines in the building site.

\section{REFERENCES}

[1] E. Gambao, C. Balaguer, A. Barrientos, R. Saltarén and E. A. Puente, "Robot Assembly System for the Construction Process Automation", IEEE International Conference on Robotics \& Automation, Albuquerque (USA), 1997.

[2] L.F. Peñín; C. Balaguer; J.M. Pastor; F.J. Rodríguez; A. Barrientos; R. Aracil; "Robotized Spraying of Prefabricated Panels", IEEE Robotics \& Automation Magazine, Sept. 1998.

[3] C. Hanser, "Fully Automated Masonry Plant", The $16^{\text {th }}$ International Symposium on Automation and Robotics in Construction, ISARC, Madrid, Spain, 1999.

[4] C Balaguer, A visit to the "SIKISUE Company in Japan"

[5] R. Diez, M. Abderrahim, V. M. Padrón, L. Celorrio, J. M. Pastor and C. Balaguer, "AUTMOD3: an Automatic 3D Modularisation System", The $17^{\text {th }}$ International Symposium on Automation and Robotics in Construction, ISARC, Taiwan 2000 